\title{
EL OPERADOR ECONÓMICO AUTORIZADO EN LOS PAÍSES DE LA COMUNIDAD ANDINA, COMO NORMA DE SEGURIDAD EN LA CADENA LOGÍSTICA DE MERCANCÍAS
}

\author{
THE AUTHORIZED ECONOMIC OPERATOR IN ANDEAN \\ COMMUNITY COUNTRIES, AS A SAFETY STANDARD \\ IN THE MERCHANDISE LOGISTIC CHAIN
}

Pablo Basantes Garcés', Guido Romero Larco², Oscar Chávez Puga², Edison Cadena

\begin{abstract}
Resumen
La seguridad en la cadena logística en el marco del programa denominado Operador Económico Autorizado (OEA), tanto de nuestro país, así como de los que conforman la Comunidad Andina (CAN) es un tema poco investigado. El objetivo de la presente investigación fue diagnosticar la situación actual del programa OEA en los países de la CAN. Para analizarlo, fue necesario comparar los beneficios que otorgan las entidades aduaneras a los OEA en cada país de la CAN, así como también fue importante consultar a las empresas exportadoras ecuatorianas sus diferentes puntos de vista frente a la implementación del programa OEA en Ecuador. Para el establecimiento del diagnóstico de los OEA se utilizó un nivel de estudio descriptivo y exploratorio con modalidad documental, y para el análisis de las empresas exportadoras del Ecuador se empleó el método cuantitativo, basado en estadistica descriptiva. Los resultados obtenidos reflejan una importante cantidad de beneficios otorgados por la entidad aduanera del Ecuador en comparación a los que otorgan las mismas entidades en los paises que conforman la CAN. En cuanto a las empresas exportadoras ecuatorianas se deduce que es poco lo que conocen en referencia al programa OEA.
\end{abstract}

\section{Palabras clave}

Seguridad en la cadena logística; Operador Económico Autorizado, Operador de Comercio Exterior y Acuerdo Reconocimiento Mutuo.

Clasificación JEL: F69

\begin{abstract}
The security in the logistics chain under the program called Authorized Economic Operator (AEO), both in our country and of those that make up the Andean Community (CAN), is a less researched topic. The purpose of the present investigation was to diagnose the current situation of the AEO program in the CAN members. To analyze this, it was necessary to compare the benefits that customs entities give to AEO in each CAN country, as well as it was important to consult Ecuadorian exporting companies for their different views in the face of implementation AEO program in Ecuador. For the establishment of the AEO diagnosis, a descriptive and exploratory study level was used with documentary modality, and the quantitative method was used for the analysis of the exporting companies of Ecuador, based on descriptive statistics. The main results obtained relate to the amount of benefits granted by the Ecuadorian customs entity compared to those granted by the same entities in the countries that make up the CAN. As far as Ecuadorian exporting companies are in terms of, little is known about the AEO program.
\end{abstract}

\section{Keywords}

Security logistics chain; Authorized Economic Operator; Foreign Trade Operator; Mutual Recognition Agreement.

JEL Classification: F69 


\section{Introducción}

Es muy poca la investigación que se ha efectuado a nivel de Operadores Económicos Autorizados (OEA) (ALADI, 2019, pág. 14) en los países que conforman la Comunidad Andina (CAN), en especial en Ecuador.

El objetivo general de esta investigación es diagnosticar la situación actual del programa de OEA en los países de la CAN. Los objetivos específicos son: comparar la situación actual en referencia a los beneficios de los OEA en los países que conforman la CAN e indagar en empresas ecuatorianas exportadoras sus posiciones frente al programa de OEA en el Ecuador.

América Latina mantiene un componente importante del comercio mundial: según el Banco Mundial en 2016 generó un PIB 5,31 billones de dólares con una población estimada en 637 millones de habitantes; tiene una serie de mecanismos que promueven el libre comercio y procesos de integración como Mercado Común Centroamericano, Comunidad del Caribe, la ya indicada Comunidad Andina, Mercado Común del Sur, originados a partir de la Asociación Latinoamericana de Integración. Por otro lado, sus países han experimentado en los últimos años impedimentos al intercambio comercial ocasionados por obstáculos a la libre circulación de mercancías provocados por instrumentos de defensa comercial y requisitos no arancelarios (Sistema Económico Latinoamericano y del Caribe, 2012, p. 13), así como otros, que tienen que ver con la implementación de procesos que aseguren la cadena logística, que se materializaron luego del 11 de septiembre de 2001, para contrarrestar los actos criminales y terroristas suscitados en Estados Unidos (Basantes \& Romero, 2018, pág. 3) a través del programa de Certificación Voluntaria de la Asociación Aduanera Comercial contra el
Terrorismo (C-TPAT por sus siglas en inglés). Posteriormente y por las mismas razones, en Europa también se concretaron programas con la misma esencia del C-TPAT como es el Marco de Estándares para Facilitar y Asegurar el Comercio (SAFE), que constituye la base del OEA que lo impulsa actualmente la Organización Mundial de Aduanas (OMA), así como iniciativas del sector privado como es la Alianza Empresarial para un Comercio Seguro (BASC, por sus siglas en inglés) o la Norma ISO 28000 (Basantes y Romero, 2018, pág. 4).

Por lo anterior, la gestión en la cadena logística se ha visto afectada, lo que ha originado dificultades en los actores del comercio internacional como son: el estado, los exportadores, los transportistas, los importadores, entre otros, que ahora además de planificar toda su operación también deben pensar en obtener certificaciones relacionadas con la cadena logística como herramienta de competitividad, que les sirva para prevenir las dificultades mencionadas, que generan sobrecostos y pérdidas de tiempo de respuesta ${ }^{5}$ (Miranda, Chamorro y Rubio, 2017, pág. 11). De acuerdo al boletín de Facilitación, Comercio y logística (FAL) de la Comisión Económica para América Latina y el Caribe (CEPAL), la seguridad logística tiene relación directa con la competitividad de una economía, por cuanto hay que agregar costos y gastos relacionados con la gestión del delito a las pérdidas directas de mercancía (2011, pág. s.p.). A esta aseveración hay que añadir que el principal beneficio para el cliente está en que los OCEs pueden garantizar de mejor manera la entrega oportuna de sus pedidos a través de la obtención de una certificación de la cadena logística.

De acuerdo a la publicación EKOS se pueden identificar 4 beneficios generales para todas las certificaciones que son:

$5 \quad$ Certificación es el proceso mediante el que una tercera parte da garantía escrita de que un producto, proceso o servicio es conforme con unos requisitos específicos. 
- Generación de oportunidades de acceso a nuevos mercados.

- Reconocimiento Institucional, producto y servicio.

- Proyección internacional.

- Logros empresariales (EKOS, 2015).

Las directrices relacionadas con la seguridad en la cadena logística implementadas por la OMA, se crearon en el 2005 a través de la implementación del Marco SAFE, que tomó cuerpo en la certificación de seguridad voluntaria de la cadena logística del comercio internacional OEA, primero en Europa y luego en las otras regiones del mundo. Este sistema parte de tres principios básicos: confianza, transparencia y cooperación entre las aduanas de los países integrantes y los diferentes operadores en la cadena logística. Dichos operadores son: los fabricantes, importadores, exportadores, agentes de aduanas, transportistas, intermediarios, operadores portuarios, aeroportuarios terminales, distribuidores, operadores integrados y de depósitos, quienes recibirían beneficios en los trámites aduaneros si habían sido calificados previamente, reforzando per se el principio que persiguen las aduanas del mundo para facilitar el comercio y generar competitividad en los países.

En junio de 2018 el Consejo de la OMA aprobó y publicó el Marco de Normas SAFE 2018. Mikuriya, K., Secretario General de la Organización Mundial de Aduanas, opina que el Marco SAFE 2018 aumenta los objetivos que tienen relación con la colaboración entre las administraciones aduaneras, a través del intercambio de información, reconocimiento de controles y asistencia administrativa mutua. Además, recomienda la cooperación con otras entidades gubernamentales que participan en el comercio de mercancías especiales como las peligrosas o el armamento o no gubernamentales como las empresas responsables de los envíos postales. Adicionalmente, prevé un listado completo de beneficios mínimos para los OEA (OMA, 2018, pág. 1).

El Marco SAFE 2018 establece cuatro elementos básicos centrales y tres pilares fundamentales sobre los cuales basa su accionar:

Primero, armoniza los requisitos de información electrónica anticipada de carga en los envíos entrantes, salientes y de tránsito. En segundo lugar, cada país que se une al Marco SAFE se compromete a utilizar un enfoque de gestión de riesgo coherente para abordar las amenazas de seguridad. En tercer lugar, requiere que como consecuencia de una solicitud razonable del país receptor, siguiendo una metodología comparable de selección de riesgo, la administración aduanera del país remitente realice una inspección saliente de la carga y/o los medios transporte de alto riesgo, preferiblemente utilizando equipo de detección no intrusivo, como aparatos de rayos $X$ a gran escala y detectores de radiación. En cuarto lugar, el Marco SAFE propone beneficios que las aduanas brindarán a las empresas que cumplan con las normas de seguridad de la cadena logística y con las mejores prácticas.

El Marco SAFE basado en los cuatro elementos centrales descritos anteriormente, se asienta en los tres pilares de los acuerdos de la red Aduanas-Aduanas, asociaciones Aduanas-Empresas y cooperación de Aduanas con otras autoridades públicas. La estrategia de tres pilares tiene muchas ventajas. Los pilares implican un conjunto de normas que se consolidan para garantizar la facilidad de comprensión y la rápida aplicación internacional (OMA, 2018, págs. 2-3).

En el Marco SAFE 2018, la OMA recomienda a las administraciones aduaneras de los países revisar los beneficios que otorgan a los OEA, clasificándolos como beneficios generales y espaciales. Dentro de los beneficios generales la OMA recomienda considerar: 
- 23 medidas para acelerar el levante de mercancías, reducir el procedimiento de tránsito y aliviar los costos de almacenamiento.

- 4 medidas de facilitación de los procedimientos después del levante.

- 4 medidas extraordinarias en caso de interrupción del comercio o nivel elevado de amenaza.

- 2 participaciones en nuevos programas/iniciativas de facilitación del comercio.

- 5 beneficios otorgados por otras autoridades públicas.

- 9 beneficios de los Acuerdos de Reconocimiento Mutuo (ARM).

- 5 información útil accesible a los participantes del OEA.

- 10 beneficios resultantes (OMA, 2018, págs. Anexo V 15-18)

Dentro de las especiales, esta entidad sugiere la adopción de una serie de ventajas para los operadores de comercio exterior, de la siguiente manera:

- 11 ventajas especiales para los exportadores

- 9 ventajas especiales para los importadores.

- 6 ventajas especiales para los agentes de almacén.

- 6 ventajas especiales para los agentes de aduana.

- 4 ventajas especiales para los operadores logísticos.

- 4 ventajas especiales para los fletadores transportistas.

- 2 ventajas especiales para los operadores portuarios de terminales.

- 2 ventajas especiales para los productores. (Organización Mundial de Aduanas, 2018, págs. Anexo V 19-20).

\section{Metodología de trabajo}

La pregunta básica de esta investigación es conocer ¿Cuál es la situación actual del programa OEA en los países de la CAN? Para contestar esta interrogante se plantearon dos preguntas específicas adicionales. ¿Cuáles son los beneficios que se otorgan a los OEA en los países que conforman la CAN? y la segunda, ¿Qué criterios mantienen las empresas exportadoras frente al programa OEA en Ecuador?

Para la primera pregunta, el nivel de estudio fue de tipo descriptivo y exploratorio, basado en un análisis documental, a partir del cual se realizó el diagnóstico de los OEA en la CAN. Para la determinación de los criterios de las empresas exportadoras del Ecuador, se efectuó un estudio de campo que tuvo como sujeto de análisis a los OCE en el Ecuador, que fueron escogidos mediante una técnica de muestreo no probabilístico, en la cual se seleccionó por conveniencia empresas exportadoras pequeñas y medianas. Según el SENAE en el Ecuador existen 47000 OCE (Moran Espin, 2014, p. xi), con la fórmula para determinar el tamaño de la muestra para población finita (Malhotra, 2008) se obtuvo un tamaño de muestra $n=$ 203 empresas exportadoras, a quienes se aplicó un cuestionario estructurado de 12 preguntas a través de una encuesta personal, que incluía preguntas con respuestas de opción múltiple, escalas de Likert, dicotómicas y abiertas.

\section{Cálculo de la muestra:}

Valor estadístico (Z) 2

$p=$ Probabilidad de Éxito; que "SI" 0,5

$q$ = Probabilidad que no ocurra el evento, es decir que "NO" 0,5

Población (N) OCE en la Ecuador 47000

Error (e) $7 \%$

Nivel de confianza: 95,5\% 


$$
\begin{gathered}
\mathrm{n}=\frac{\mathrm{Z}^{2} * P * Q * N}{\mathrm{E}^{2} *(\mathrm{n}-1)+\mathrm{Z}^{2} * \mathrm{P} * \mathrm{Q}} \\
\mathrm{n}=\frac{(2)^{2} * 0.5 * 0.5 * 4700}{(0.007) *(4700-1)+\left((2)^{2} * 0.5 * 0.5\right)} \\
\mathrm{n}=\frac{4700}{231.30} \\
\mathrm{n}=203
\end{gathered}
$$

\section{Resultados}

¿Cuál es la situación actual del programa OEA en los países de la CAN?

En la tabla 1 se puede observar que el país que más operadores OEA calificados en
América del Sur tiene es Brasil con 84, le sigue Uruguay con 33, Colombia con 23 y Perú con 21, mientras que Ecuador tiene 5. De entrada, nuestros vecinos directos y miembros de la CAN tienen un mayor número de OEA calificados.

Tabla 1. Situación OEA en países de América del Sur

\begin{tabular}{|l|l|l|l|}
\hline \multicolumn{1}{|c|}{ Países } & Año implementación & \multicolumn{1}{|c|}{ Tipo de Operadores } & Operadores calificados \\
\hline Argentina & 2006 & $\begin{array}{l}\text { Importadores } \\
\text { Exportadores }\end{array}$ & 7 \\
\hline Bolivia & 2015 & $\begin{array}{l}\text { Exportadores } \\
\text { Agentes de aduanas } \\
\text { Importadores } \\
\text { Transportistas }\end{array}$ & 4 \\
\hline Brasil & 2014 & $\begin{array}{l}\text { Importadores } \\
\text { Exportadores }\end{array}$ & 84 \\
\hline Colombia & 2011 & $\begin{array}{l}\text { Importadores } \\
\text { Exportadores }\end{array}$ & 23 \\
\hline Ecuador & 2015 & $\begin{array}{l}\text { Exportadores } \\
\text { Perú }\end{array}$ & $\begin{array}{l}\text { Importadores } \\
\text { Exportadores }\end{array}$ \\
\hline Uruguay & 2012 & $\begin{array}{l}\text { Importadores } \\
\text { Exportadores } \\
\text { Agentes de aduana } \\
\text { Transportistas terrestres } \\
\text { Usuarios de zonas francas } \\
\text { Almacenes transitorios } \\
\text { Operadores de puerto } \\
\text { Terminales de carga }\end{array}$ & 33 \\
\hline Chile & 2017 & $\begin{array}{l}\text { Exportadores } \\
(*)\end{array}$ \\
\hline
\end{tabular}

${ }^{\star}$ ) En marcha al momento de la recopilación de la información

Fuente: AEO Compendio 2017 (Organización Mundial de Aduanas, 2017) 
El proceso de certificación OEA en Ecuador inició en el año 2015. En febrero de 2018 mediante Decreto Ejecutivo 312 el gobierno ecuatoriano declaró al programa OEA como Política para la Facilitación del Comercio Exterior (Basantes y Romero, 2018, pág. 7). Esta certificación ayuda a las empresas que efectúan comercio internacional a ganar mayor competitividad. La dinamización del sector exportador es clave para una economía dolarizada: el estado promueve el acceso a nuevos mercados para su oferta exportable y consolida relaciones de reciprocidad en el ámbito de comercio internacional (Secretaría Nacional de Planificación y Desarrollo, 2017, pág. 73). Por lo anterior, es prioritario conocer los criterios de las empresas exportadoras del país frente a la implementación del programa OEA, con el ánimo de recomendar acciones que incrementen el número de empresas calificadas.

En la actualidad existe una gran variedad de certificaciones de gestión de calidad, medioambientales, prevención de riesgos laborales, responsabilidad social, seguridad en la cadena logística, entre otras (Miranda, Chamorro y Rubio, 2017, pág. 6), que han despertado el interés de los operadores de comercio exterior, obligándoles a identificar y escoger según sus necesidades la más beneficiosa para sus actividades. Según Guz- mán (2016), indica que los países con gran número de OEA como Estados Unidos y China prefieren realizar negociaciones con empresas de países que compartan las mismas iniciativas.

El programa OEA busca generar para las empresas ventajas competitivas, seguridad en el transporte y una reducción de los tiempos de entrega de las mercancías (Inter-American Development Bank, 2018). En el ámbito regional el programa se ha desarrollado desde 2006 en Argentina y 2011 en Colombia, país integrante de la CAN.

Para establecer la situación actual del programa OEA a nivel de los países CAN es necesario diagnosticar los beneficios que ofrece cada miembro.

En la tabla 2 se puede observar que Bolivia es el país de la CAN que más beneficios brinda a sus OEA, llegando en total a 42 , donde 10 son para todos los tipos de operadores, 12 para importadores y exportadores, 5 para despachantes de aduana, 9 para transportistas terrestres y 5 para otras entidades de control. Sin embargo, de lo anterior el número total de OEA en ese país hasta el 2017 alcanza 4 operadores. Colombia con 37 beneficios ofertados certificó a 23 operadores, Perú con 29 certificó a 21 operadores, y Ecuador con 9 beneficios calificó a 5 operadores, todos contados hasta 2017.

Tabla 2. Cantidad de Beneficios OEA países CAN

\begin{tabular}{|l|l|l|l|l|}
\hline \multicolumn{1}{|c|}{ País } & Año de implementación & $\begin{array}{l}\text { Operadores } \\
\text { calificados }\end{array}$ & $\begin{array}{l}\text { Tipo de operadores } \\
\text { calificados }\end{array}$ & $\begin{array}{c}\text { No. de beneficios } \\
\text { operadores }\end{array}$ \\
\hline Colombia & 2011 & 23 & Exportadores & 37 \\
\hline Perú & 2012 & 21 & Operadores & 31 \\
\hline Ecuador & 2015 & 5 & $\begin{array}{l}\text { Exportadores y Agen- } \\
\text { te de Aduanas }\end{array}$ & 8 \\
\hline Bolivia & 2015 & 4 & $\begin{array}{l}\text { Exportadores } \\
\text { Agentes de Aduana } \\
\text { Importadores } \\
\text { Transportistas }\end{array}$ & 42 \\
\hline
\end{tabular}

Fuente: (Aduana Nacional Bolivia, 2017) (DIAN, 2016) (SUNAT Perú, 2017) (SENAE, 2017) 
En la Tabla 3 se hace un análisis de los principales de beneficios que otorgan los países de la CAN. El beneficio que más se repite, tiene que ver con la asignación de un funcionario al operador en los trámites que va a efectuar, otro beneficio común está relacionado con el modo de realización de los aforos, la priorización en los tiempos de atención a las declaraciones de los operadores, y en menor medida la reducción de garantías aduaneras y la reducción o nulidad de los aforos físicos. En Colombia existe un beneficio que no es general para el resto de los países, se puede efectuar desaduanamientos de las mercancías en las instalaciones del declarante.

Tabla 3. Principales Beneficios OEA países CAN

\begin{tabular}{|c|c|c|c|}
\hline Bolivia & Colombia & Perú & Ecuador \\
\hline $\begin{array}{l}\text { Asignación de un funciona- } \\
\text { rio aduanero }\end{array}$ & Asignación de un oficial & Consultas técnicas & Atención personalizada \\
\hline Reducción de garantías & Reducción de garantías & N/A & $\mathrm{N} / \mathrm{A}$ \\
\hline $\begin{array}{l}\text { Aforo en planta con la verifi- } \\
\text { cación de forma remota del } \\
\text { embarque y colocación de } \\
\text { precintos }\end{array}$ & Inspección no intrusiva & $\begin{array}{l}\text { Cero reconocimientos fí- } \\
\text { sicos a importaciones y } \\
\text { exportaciones }\end{array}$ & $\mathrm{N} / \mathrm{A}$ \\
\hline $\begin{array}{l}\text { Priorización de atención (Au- } \\
\text { torizaciones previas - des- } \\
\text { pacho aduanero) }\end{array}$ & N/A & N/A & $\begin{array}{l}\text { Reducción de tiem- } \\
\text { pos y costos (Facilitar } \\
\text { tramitación) }\end{array}$ \\
\hline N/A & $\begin{array}{l}\text { Realiza desaduana mien- } \\
\text { to de las mercancías ob- } \\
\text { jeto de importación en } \\
\text { las instalaciones del de- } \\
\text { clarante. (M) }\end{array}$ & N/A & $\mathrm{N} / \mathrm{A}$ \\
\hline
\end{tabular}

Fuente: (Aduana Nacional Bolivia, 2017) (DIAN, 2016) (SUNAT Perú, 2017) (SENAE, 2017)

En la tabla 4 se puede observar la relación de la aduana con las entidades gubernamentales de cada país con las cuales mantiene convenios internos, se tiene que: en Colombia, en la reglamentación que regula el programa OEA en ese país suscrita por la Dirección de Impuestos y Aduanas Nacionales DIAN, ya está claramente explícita la interrelación entre las entidades internas (2016), lo propio se puede observar en la normativa que comunica los beneficios que se pueden obtener con la calificación OEA de la entidad aduanera de Bolivia, donde se especifica incluso el convenio interinstitucional vigente con dichos organismos (Aduana Nacional Bolivia, 2017).
Al revisar el documento de beneficios que otorga la entidad aduanera de Perú a los OEA, se constata que solo hay una referencia en cuanto a ventajas con otras entidades gubernamentales. En el caso de Ecuador, el Decreto Ejecutivo 312 que declaró al programa OEA como parte de la Política de Facilitación al Comercio Exterior, obliga a otras entidades del estado involucradas con el comercio exterior ecuatoriano a implementar, desarrollar y mantener operativo el programa OEA. Estas entidades son: Ministerio del Interior, la Agencia Nacional de Regulación, Control y Vigilancia Sanitaria ARCSA, Agencia Ecuatoriana de Aseguramiento de Calidad del Agro AGROCALIDAD, Ministerio de Transporte y Obras Públicas, y demás en- 
tidades de apoyo de acuerdo a la ejecución del programa, todas ellas funcionando en el portal de la Ventanilla Única Ecuatoriana (VUE) (SENAE, 2018).

Tabla 4. Convenios con entidades internas de cada país

\begin{tabular}{|l|l|l|l|}
\hline \multicolumn{1}{|c|}{ BOLIVIA } & \multicolumn{1}{|c|}{ COLOMBIA } & \multicolumn{1}{c|}{ PERÚ } & \multicolumn{1}{c|}{ ECUADOR } \\
\hline $\begin{array}{l}\text { SEVANEX } \\
\text { Servicio Nacional de } \\
\text { Exportaciones }\end{array}$ & $\begin{array}{l}\text { Policía Nacional de } \\
\text { Colombia }\end{array}$ & $\begin{array}{l}\text { IAMC } \\
\text { Intendencia de Aduana } \\
\text { Marítima del Callao }\end{array}$ & $\begin{array}{l}\text { VUE } \\
\text { Ventanilla Única } \\
\text { Ecuatoriana }\end{array}$ \\
\hline $\begin{array}{l}\text { IBMETRO } \\
\text { Instituto Boliviano de } \\
\text { Metrología }\end{array}$ & Autoridad Sanitaria ICA & & \\
\hline $\begin{array}{l}\text { ASP-B } \\
\text { Administración de Servi- } \\
\text { cios Portuarios }\end{array}$ & $\begin{array}{l}\text { Autoridad Fitosanitaria } \\
\text { INVIMA }\end{array}$ & & \\
\hline $\begin{array}{l}\text { SENASAG } \\
\text { Servicio Nacional de Sani- } \\
\text { dad Agropecuaria }\end{array}$ & $\begin{array}{l}\text { Ministerio de Comercio } \\
\text { Exterior }\end{array}$ & & \\
\hline $\begin{array}{l}\text { IBTEN } \\
\text { Instituto Boliviano de } \\
\text { Ciencia y Tecnología } \\
\text { Nuclear }\end{array}$ & & & \\
\hline
\end{tabular}

Fuente: (Aduana Nacional Bolivia, 2017) (SENAE, 2018) (DIAN, 2016) (SUNAT Perú, 2017)

De la revisión efectuada a los beneficios presentados por las diferentes entidades de aduana de los países de la CAN se puede destacar lo siguiente:

Si analizamos el entorno OEA-CAN podemos evidenciar que el beneficio común de aplicaciones radica en adoptar un representante OEA para solucionar y verificar las operaciones que realicen los operadores OEA en la cadena logística. De este modo los OEA e interesados podrán solicitar información, asesoría, consultas técnicas para un correcto desarrollo del programa, y a su vez, el representante podrá rendir cuentas y solicitar otros beneficios a los representantes gubernamentales dependiendo la necesidad de los OEA y de su alcance.

A fin de otorgar mayores incentivos $y$ beneficios a los OEA, se ha aplicado la reducción de garantías aduaneras, siendo este un gran incentivo para los importadores, y así reduciendo los costos a su cadena logística, generando mayor utilidad, y a su vez siendo un programa más llamativo para los actores de comercio exterior.

Los países miembros de la CAN (excepto Ecuador) han decidido eliminar o modificar los aforos físicos, con ello se logra una reducción de tiempos y costos generando así valor agregado a sus operaciones dentro de la cadena logística.

Las aplicaciones del programa OEA en Ecuador, se encuentran en un contexto muy generalizado. No se especifica el desarrollo de estas, como, por ejemplo: ¿cómo logrará reducir costos y tiempos en las operaciones de los OEA?

De igual manera, se habla de facilitar la tramitación de las operaciones de comercio exterior, pero no da a detalle del cómo se va a efectuar, o qué incentivos da el Ecuador para que los OCE accedan al programa OEA. 
Países como Colombia, además de lo antes mencionado, otorga la devolución del IVA por dos meses, a fin de que el programa tenga una mayor acogida.

\section{Los Acuerdos de Reconocimiento Mutuo}

El 10 de febrero de 2017, en la Secretaría General de la CAN ubicada en Lima (Perú), se llevó a cabo la primera reunión para la el establecimiento de un plan de acción para lograr Acuerdos de Reconocimiento Mutuo (ARM) entre las aduanas de los países miembros de este bloque (Comunidad Andina, 2018), que culminaron finalmente con la firma del ARM entre los países que conforman la CAN en mayo de 2019 (SENAE, 2019).

Por otra parte, Colombia y Perú tienen adelantado otros ARM con el bloque de países de la Alianza del Pacífico, que está conformada por: Colombia, Perú, Chile y México. Para este bloque comercial definieron un plan de trabajo compuesto por cuatro etapas. Hasta marzo de 2017 estaban cumplidas dos de las 4 etapas planificadas (DIAN Colombia, 2017).

El Decreto Ejecutivo 312 también, da paso para que el SENAE promueva la consecución de Acuerdos de Reconocimiento Mutuo con las aduanas de otros países (SENAE, 2018, pág. 9). De la misma manera en octubre de 2018 la Asamblea Nacional de Ecuador aprobó el Protocolo de Enmienda al Acuerdo sobre Facilitación al Comercio en el marco de la Organización Mundial de Comercio (OMC).

El Ministerio de Producción, Comercio Exterior e Inversiones promovió la aprobación de este instrumento con el objeto de agilizar los procesos y trámites de exportación e importación; optimizar los procesos aduaneros y las políticas implementadas por las distintas instituciones gubernamentales relacionadas con el comercio; así como fortalecer la institucionalidad ecuatoriana a través de asistencia técnica y cooperación financiera (Ministerio de Comercio Exterior, 2018).

El SENAE inició el programa OEA en agosto de 2015 con la calificación de 5 empresas. Posteriormente, a finales de 2017 este se suspendió. De acuerdo al portal del SENAE, en abril de 2018 se presentó una repotenciación del programa, y no es sino hasta agosto de 2019 que la entidad aduanera del Ecuador promovió la Postulación en Línea (SENAE, 2019). Es incuestionable la relegación que ha tenido el programa en el país, en este último periodo presidencial desde mayo de 2016 tres directores generales han pasado ya por la institución, lo cual ha dificultado su sostenimiento y desarrollo.

Para responder a la pregunta ¿Qué criterios mantienen las empresas exportadoras frente al programa OEA en Ecuador?, se efectuaron encuestas donde se obtuvo la siguiente información:

\section{Encuestas a empresas exportadoras del Ecuador}

\section{Sectores de exportación de empresas encuestadas}

En la Figura 1 se puede observar que el 59\% de las empresas exportadoras encuestadas se dedican a exportar productos manufacturados, el $36 \%$ a la exportación corresponde a productos agropecuarios, el $4 \%$ restante a la rama de combustibles y productos de industria extractiva. 
Figura 1. Sectores de exportación de empresas encuestadas

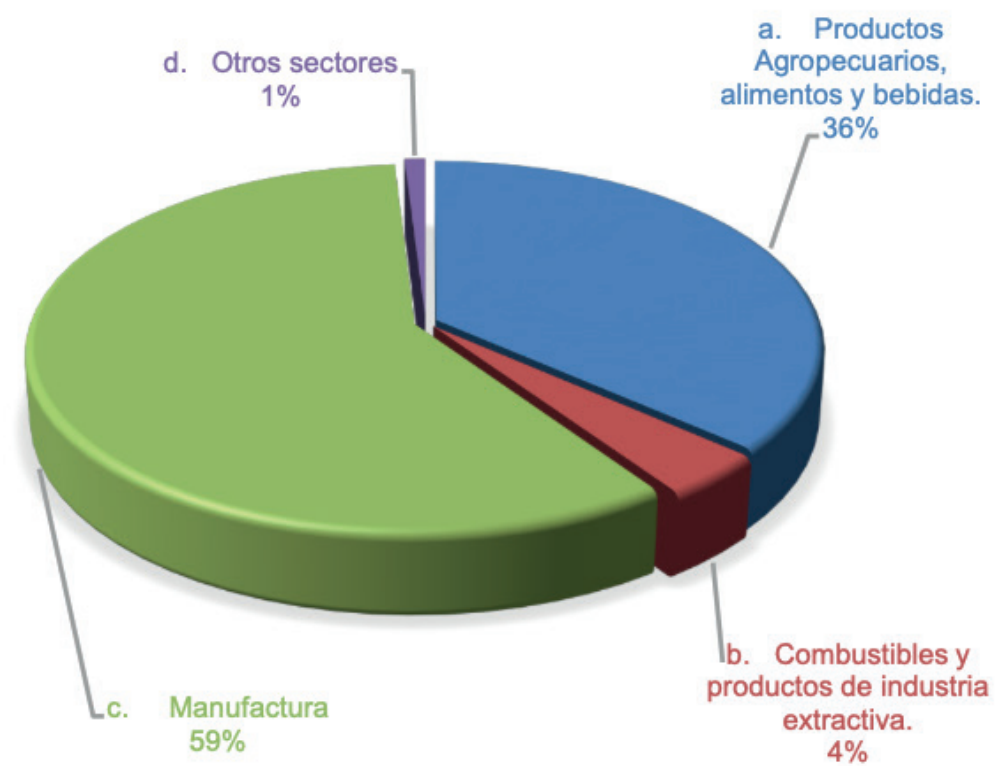

a. Productos Agropecuarios, alimentos y bebidas.

ab. Combustibles y productos de industria extractiva.

ac. Manufactura

ad. Otros sectores

Fuente: Encuesta a empresas exportadoras del Ecuador.

\section{Periodicidad de las exportaciones}

En la Figura 2 se puede observar que el $72 \%$ de los encuestados efectúan expor- taciones regulares durante el año, el 18\% trimestrales, el $7 \%$ semestrales y el $2 \%$ anuales

Figura 2. Periodicidad de las exportaciones

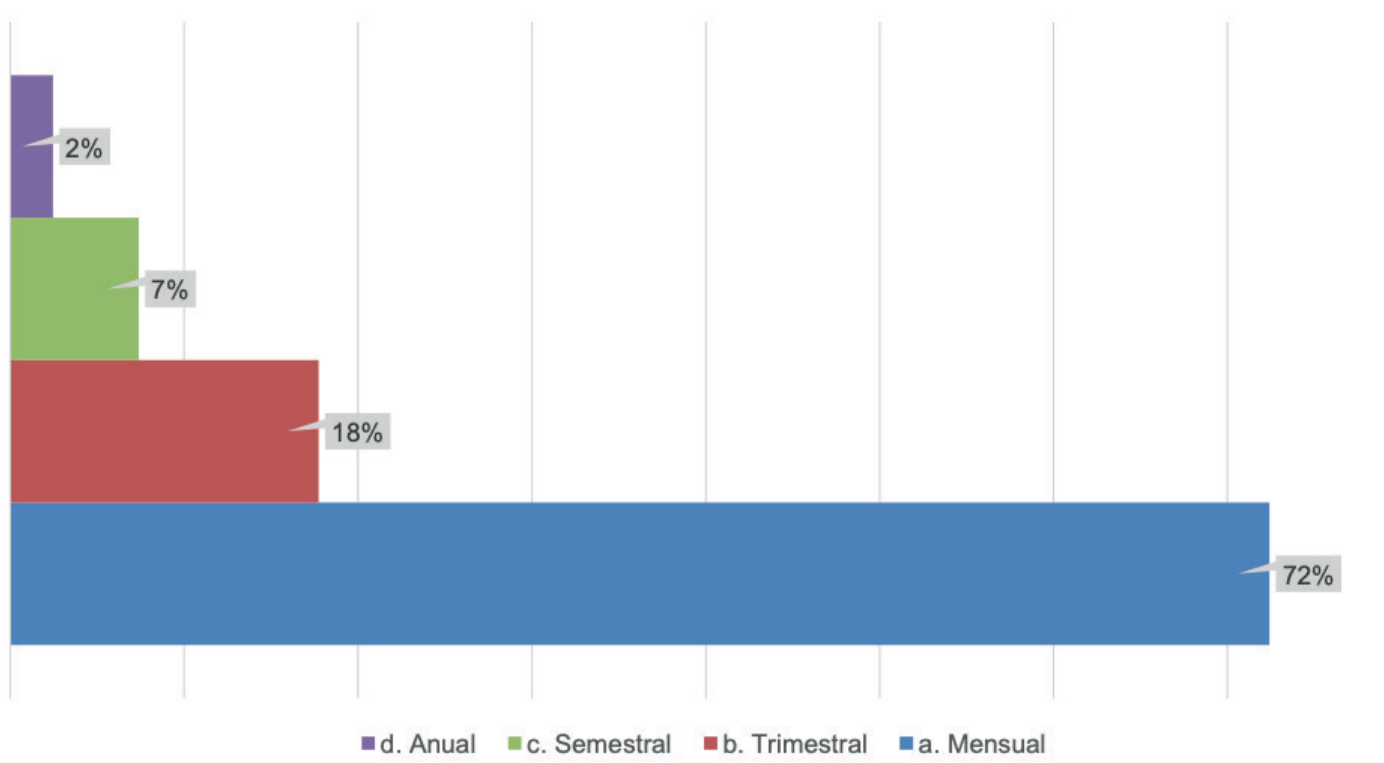

Fuente: Encuesta a empresas exportadoras del Ecuador. 


\section{Cumplimiento de Requisitos legales para calificarse como OEA}

De acuerdo con la Figura 3 se puede verificar que el $80 \%$ de las empresas exportadoras encuestadas creen que cumplen con la normativa requerida por la autoridad aduanera para calificarse como OEA en Ecuador.

Figura 3. Empresas que cumplen requisitos legales para calificarse como OEA

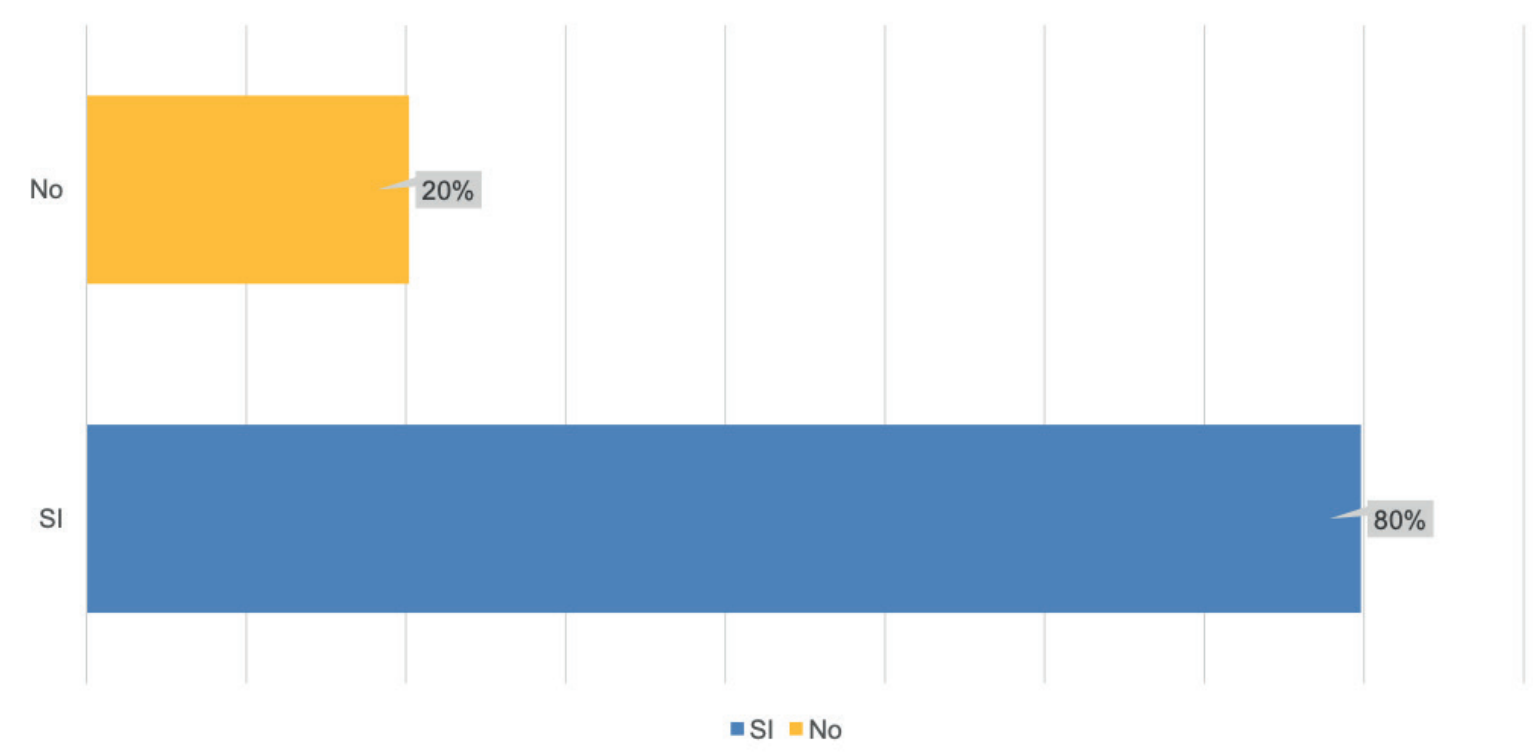

Fuente: Encuesta a empresas exportadoras del Ecuador.

\section{Razones para calificarse como OEA}

De acuerdo a la Figura 4 la principal razón por la cual buscarían las empresas exportadoras encuestadas calificarse como OEA, es para ser más competitivos a nivel internacional con un $28 \%$, buscan también facilidades en los trámites aduaneros en un $27 \%$, les interesa además, los beneficios que contraen los Acuerdos de Reconocimiento Mutuo entre entidades aduaneras de los diferentes países en un 16\%, la aten- ción personalizada permanente con el área OEA les interesa a un $15 \%$, y el reconocimiento con otras entidades del estado les parece interesante a un $14 \%$.

\section{Conocimiento de las empresas sobre la certificación OEA en el Ecuador}

En la Figura 5 se puede observar que el 33\% de las empresas exportadoras encuestadas sí conocen sobre la certificación OEA en el Ecuador, mientras que el $67 \%$ lo desconoce. 
Figura 4. Razones para calificarse como OEA empresas exportadoras Ecuador

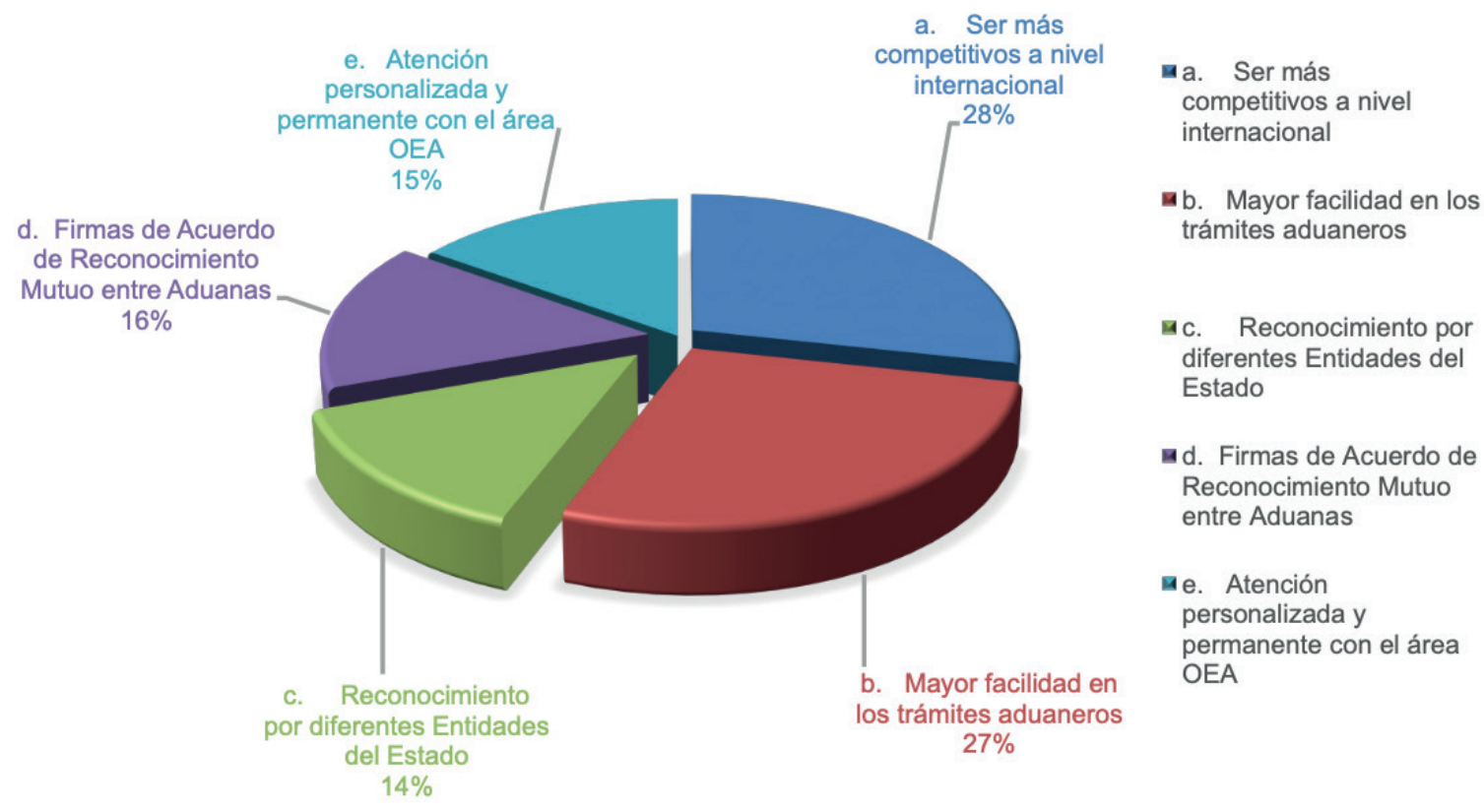

Fuente: Encuesta a empresas exportadoras del Ecuador.

Figura 5. Conocimiento de las empresas exportadoras sobre el programa OEA en Ecuador

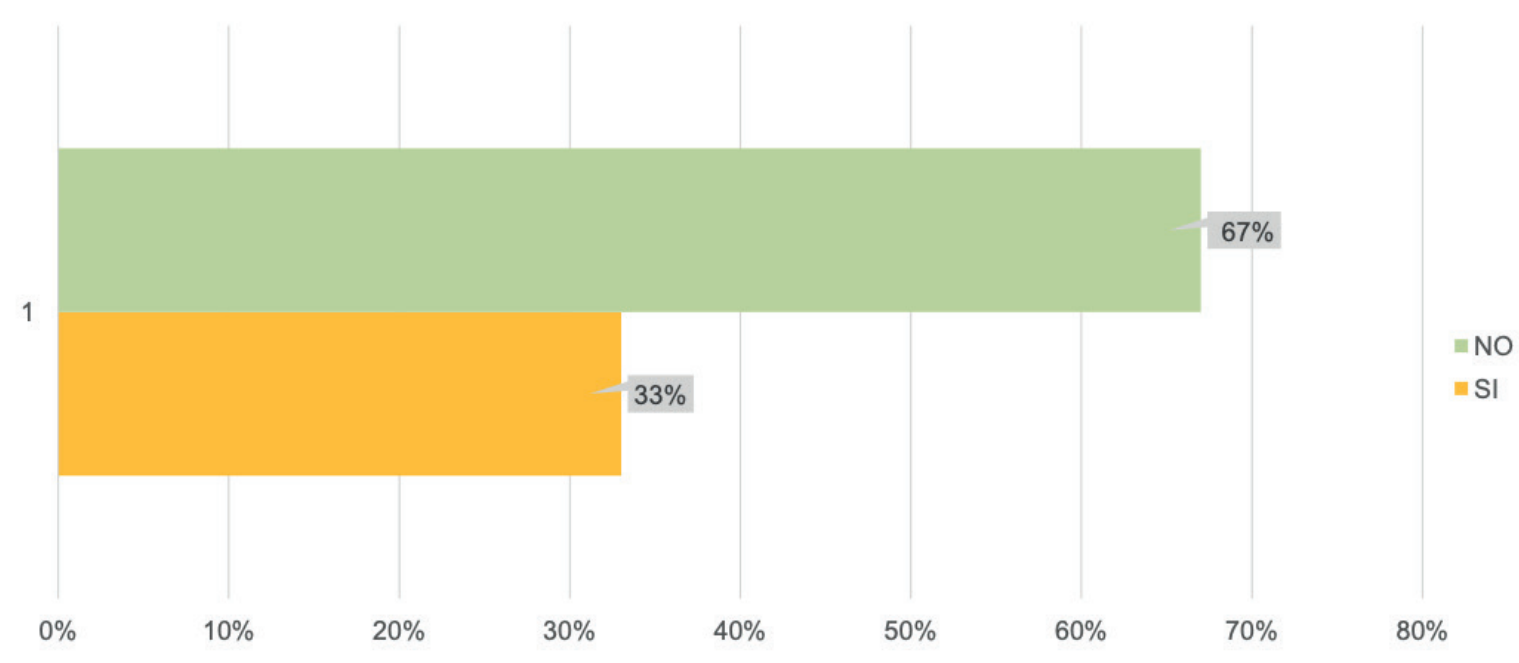

Fuente: Encuesta a empresas exportadoras del Ecuador. 


\section{Certificaciones de las empresas exportadoras ecuatorianas}

En la Figura 6 se puede observar que el 53\% de las empresas exportadoras encuestadas no disponen de algún tipo de certificación logística internacional, mientras que el 47\% sí disponen y no consideran necesario calificarse como OEA.

Figura 6. Certificaciones de las empresas exportadoras ecuatorianas

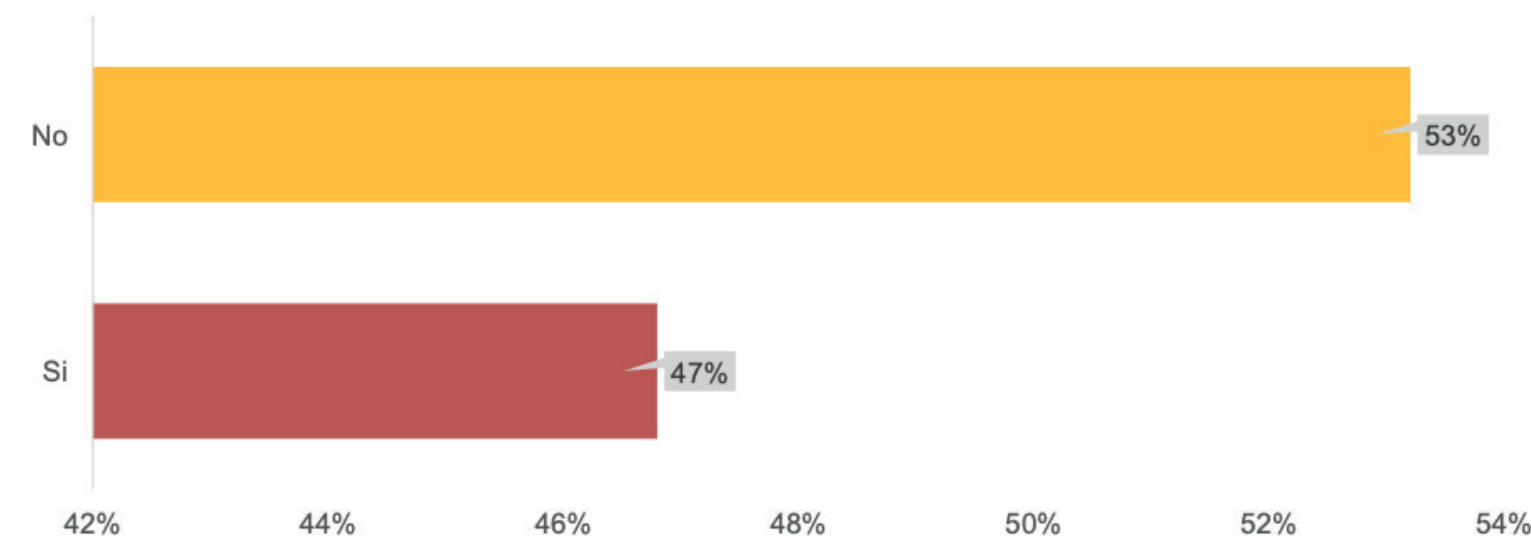

Fuente: Encuesta a empresas exportadoras del Ecuador.

\section{Certificaciones internacionales de las empresas exportadoras ecuatorianas}

La Figura 7 indica que la mayor certificación internacional que poseen las empresas ex- portadoras en el país es la BASC con un $66 \%$, le sigue la ISO 28000 con un 22\%, a continuación, está la certificación SAFE con un $9 \%$ y por último la CTPAT con un 3\%.

Figura 7. Certificaciones Internacionales que poseen las empresas exportadoras

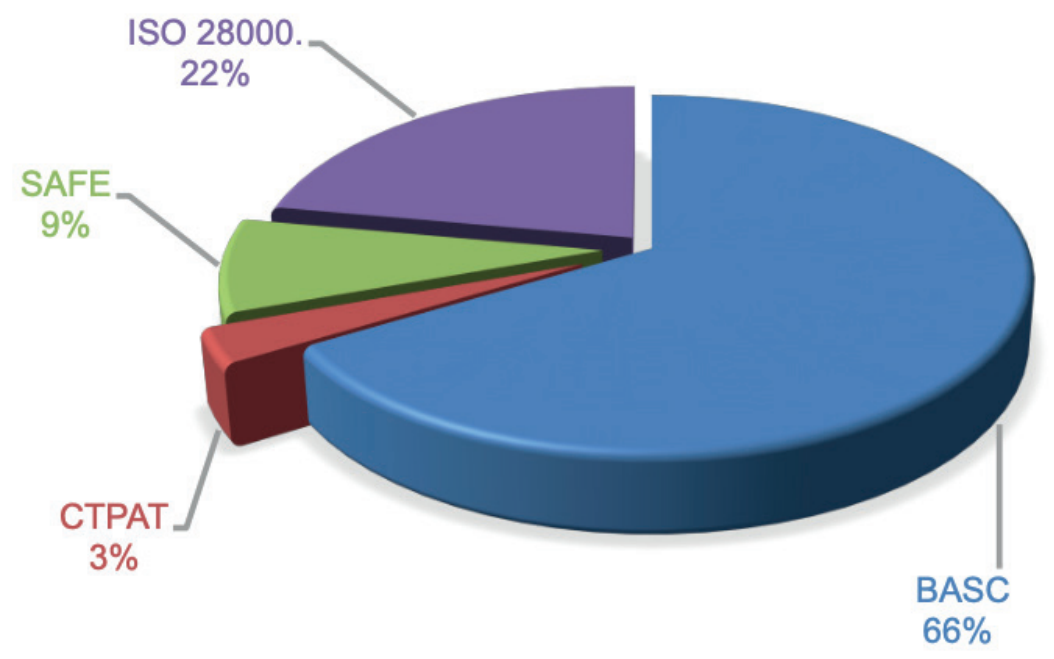

BASC

a CTPAT

口SAFE

ISO

28000.

Fuente: Encuesta a empresas exportadoras del Ecuador. 


\section{Razones de las empresas exportadoras ecuatorianas para no calificarse OEA}

La Figura 8 resalta las principales razones por las cuales las empresas encuestadas no consideran importante la calificación en el marco del Programa OEA en el Ecuador. La principal de todas es que el SENAE no ha puesto énfasis para que las empresas busquen la calificación con un 46\%, Consideran además que el proceso para la obtención de la certificación es complicado un 23\%. No tienen confianza en las certificaciones emitidas por el SENAE un 10\% y tienen inseguridad de que la certificación OEA de Ecuador les sirva en otros países un 10\%.

Figura 8. Razones de las exportadoras para no calificarse como OEA.

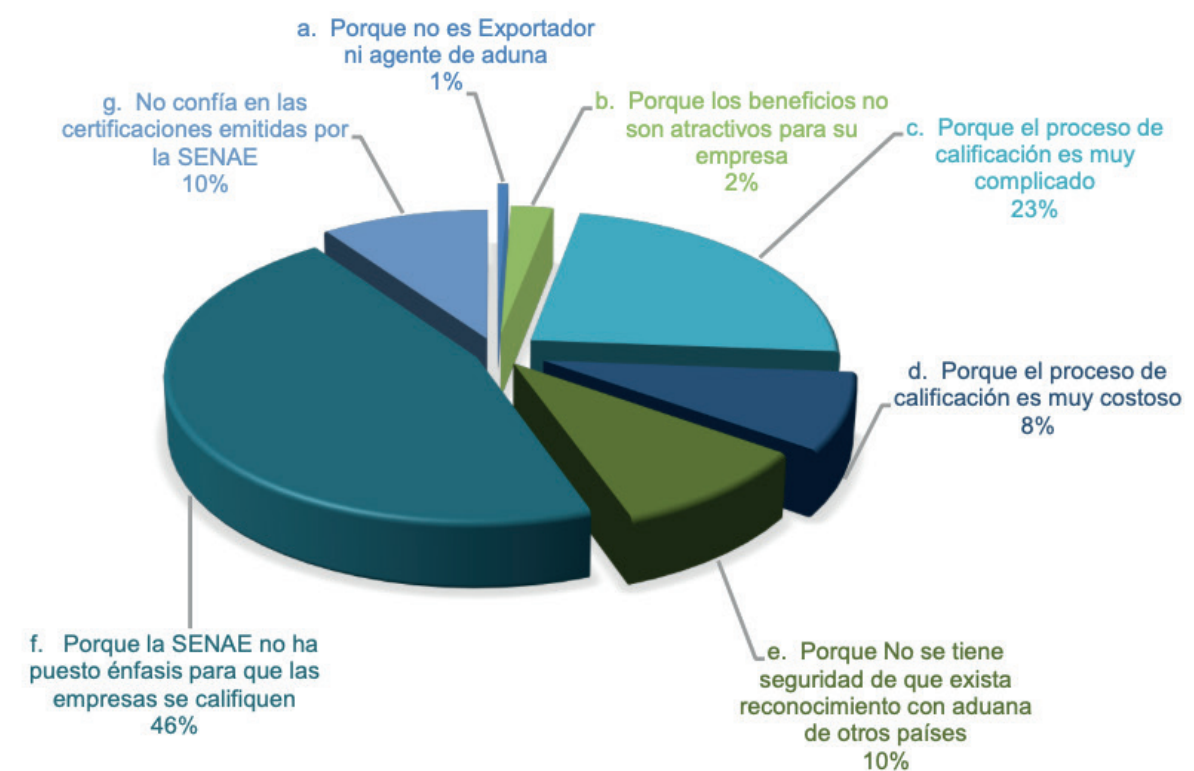

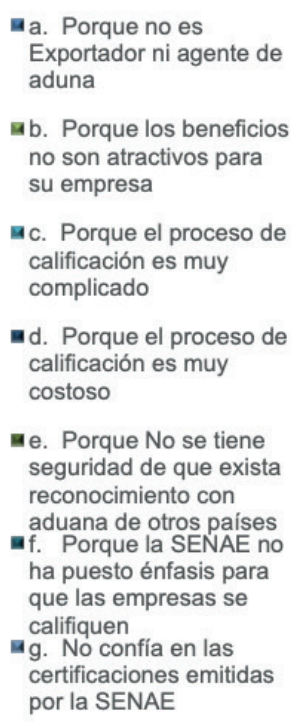
no son atractivos para su empresa

c. Porque el proceso de calificación es muy complicado

ad. Porque el proceso de calificación es muy costoso

a. Porque No se tiene seguridad de que exista reconocimiento con aduana de otros paises f. Porque la SENAE no ha puesto énfasis para que las empresas se califiquen

g. No confía en las certificaciones emitidas por la SENAE

Fuente: Encuesta a empresas exportadoras del Ecuador.

\section{Presupuesto de las empresas exportadoras para la obtención de certificación OEA}

La Figura 9 muestra que el 69\% de las empresas exportadoras encuestadas conside- ran que el valor que invertirían para obtener una certificación OEA oscila entre los USD 600 y los USD 1 000. El 30\% estaría dispuesto a invertir entre USD 1000 y los USD 2 000. Finalmente, un 1\% investiría entre USD 2000 y USD 3000 
Figura 9. Presupuesto estimado de las empresas exportadoras para la certificación OEA

c. 2000 a 3000 USD

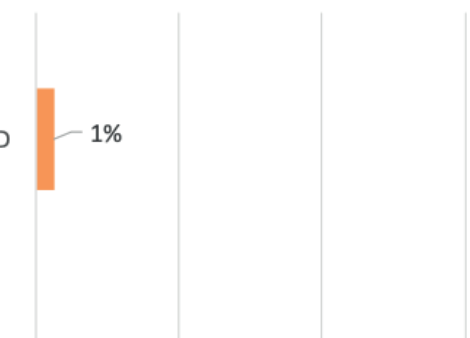

b. 1000 a 2000 USD

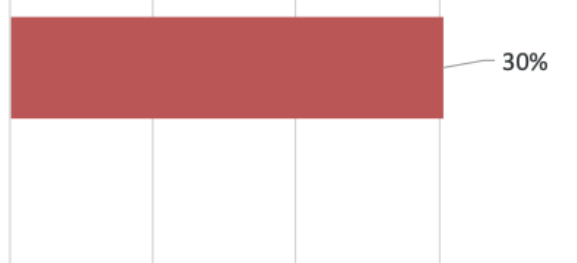

a. 600 a 1000 USD

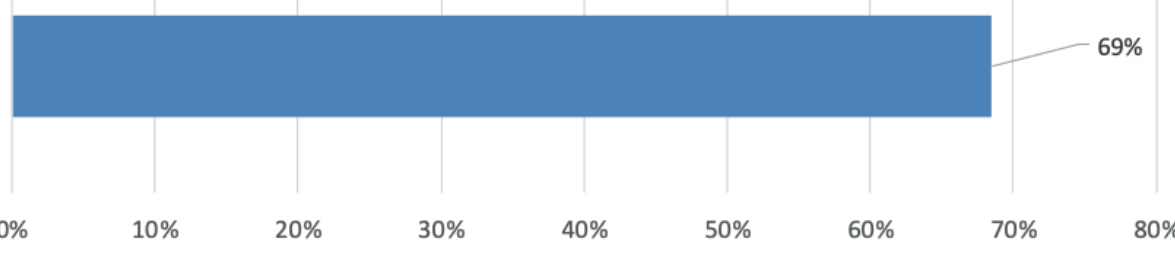

Fuente: Encuesta a empresas exportadoras del Ecuador.

\section{Disposición de tiempo a invertir para calificarse como OEA}

La Figura 10 establece que el $57 \%$ de las empresas encuestadas estarían dispues- tas a invertir máximo entre 10 a 30 días de tiempo para calificarse como OEA, el 24\% invertirían entre 30 y 60 días y el 19\% entre 60 y 90 días.

Figura 10. Tiempo por invertir para calificarse como OEA

c. 60 días a 90 días

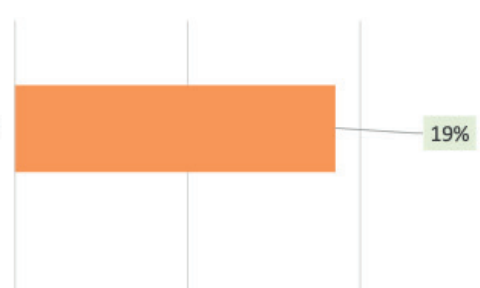

b. 30 días a 60 días

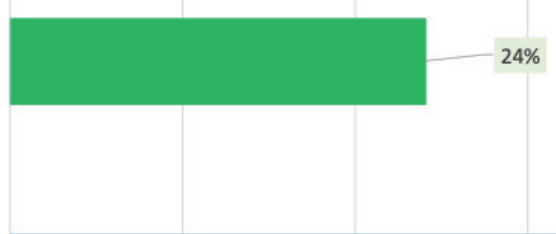

a. 10 días a 30 días

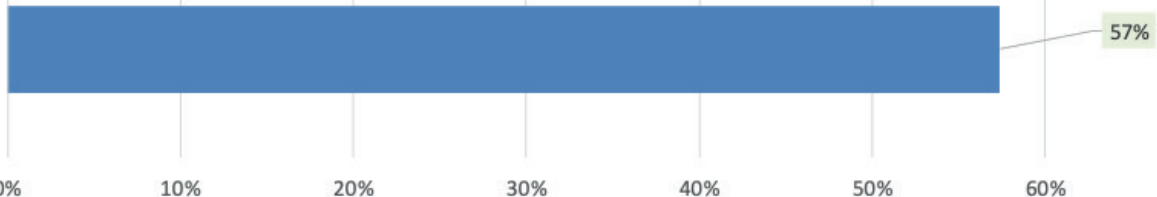

$0 \%$

$10 \%$

$60 \%$

$70 \%$

Fuente: Encuesta a empresas exportadoras del Ecuador. 


\section{Conclusiones}

Sobre la situación actual de los OEA en los países de la CAN se puede concluir lo siguiente:

A la fecha de la realización de la investigación Ecuador alcanzó a certificar solo cinco empresas, el único país de la CAN que está por debajo del nuestro es Bolivia que obtuvo cuatro, le sigue Perú y con 21 y Colombia con 23.

Los beneficios ofertados en Ecuador para los OEA son mínimos en comparación con los propuestos por otros países de la CAN. Adicionalmente los beneficios planteados para Ecuador son generales, mientras que los ofrecidos por los otros países de la CAN llegan a niveles operativos inclusive, y podrían generar ventajas competitivas a nivel internacional porque ayudarían a los OCE a tener mayor facilidad en los trámites aduaneros en origen y destino, generando ahorros de tiempo y costo.

Si lo que Ecuador busca es el desarrollo del programa OEA, es fundamental la firma y ejecución de los ARM con los países de la CAN y con otros bloques comerciales. La firma de estos convenios están supeditados a beneficios mutuos entre los países actuantes, para Ecuador la mayor importancia está en el fomento a las exportaciones, motivo por el cual en primer término se persigue la certificación para los exportadores, más sin embargo, los países con los que firmaríamos el reconocimiento mutuo también persiguen objetivos de exportación, que para nosotros serían importaciones, por tanto, también es fundamental que se incorpore a los importadores como potenciales certificados OEA, de modo que sea atractiva la firma de los ARM para los otros países. Por otra parte, es incuestionable la relegación que ha tenido el programa OEA en Ecuador, desde mayo de 2016 a la fecha tres directores generales han pasado ya por la institución, lo cual ha dificultado su sostenimiento y desarrollo.
En lo referente a los criterios de las empresas exportadoras sobre la implementación del programa OEA en Ecuador, se establece que el mayor problema que existe es el desconocimiento que tienen del mismo, por cuanto lo conocen apenas en un 33\%.

El $47 \%$ de los encuestados disponen de una certificación logística internacional y no consideran necesario calificarse como OEA. La certificación logística internacional que más poseen las empresas del sector es la BASC con un 66\%. La principal razón de las empresas para no buscar una certificación OEA es que el $46 \%$ consideran que el SENAE no ha puesto el énfasis necesario para impulsar el programa, además de que consideran complicado el proceso de obtención en un $23 \%$, no tienen confianza en las certificaciones emitidas por el SENAE un 10\%, y tienen inseguridad de que la certificación OEA les pueda servir en otros países en un 10\%. El 69\% de empresas encuestadas consideran que el valor que invertirían en buscar la certificación de OEA debe oscilar entre los USD 600 y USD 1000, y el 57\% estaría dispuesto a destinar entre 10 y 30 días para calificarse. La principal causa por la cual las empresas exportadoras consideran que podrían buscar la certificación OEA es para ser más competitivos a nivel internacional, así lo reconocen el $28 \%$ de las empresas encuestadas, mientras que un $27 \%$ optarían por la certificación para obtener beneficios al momento de efectuar los trámites aduaneros. Bajo todo este contexto, el papel de la entidad aduanera es fundamental para desarrollar el programa, se debe trabajar más sobre mejorar los beneficios de calificarse como OEA. El SENAE debe orientar a los OCE a realizar una evaluación costo - beneficio de la certificación OEA, porque si bien ésta no tiene costo por ser de carácter gubernamental, si exige adecuaciones administrativas y control, elementos que junto al mantenimiento quizás no genere los réditos a corto plazo, 
pero sus actividades de comercio exterior obtendrán un valor agregado por la competitividad de la empresa.

\section{Bibliografía}

Aduana Nacional Bolivia. (2017). Beneficios del Operador Económico Autorizado. Recuperado el 26 de 11 de 2019, de https://www. aduana.gob.bo/oea/sites/default/files/ Documentos/B02.\%20BENEFICIOS_OEA_ CONSOLIDADO_V1.pdf

ALADI. (26 de marzo de 2019). Estudio sobre los programas operador económico autorizado en los países. Montevideo - Uruguay: ALADI Secretaría General. Obtenido de http://www2.aladi.org/biblioteca/Publicaciones/ALADI/Secretaria_General/SEC_ Estudios/229rev1.pdf

Basantes, P., y Romero, G. (2018). Diagnóstico de los Operadores Económicos Autorizados (OEA) en el Ecuador. Revista Economía y Negocios, 9 num 1, 11. Obtenido de https:// revistas.ute.edu.ec/index.php/economia$y$-negocios/article/view/440/380

CEPAL. (2011). Seguridad de la cadena logística y su incidencia en la competitividad de Mesoamérica. Obtenido de https:// repositorio.cepal.org/bitstream/handle/11362/36084/1/FAL-300-WEB_es.pdf

Comunidad Andina. (15 de septiembre de 2018). Acuerdo de reconocimiento de los programas OEA CAN. Obtenido de http://www. comunidadandina.org/Prensa.aspx?id=3 886\&accion=detalle\&cat $=$ NP\&title $=$ pais es-de-la-comunida

DIAN. (1 de enero de 2016). Beneficios Del Operador Económico Autorizado Colombia. Obtenido de Https://www.dian.gov.co/ aduanas/oea/documents/beneficios\%20 oea.pdf

DIAN Colombia. (16 de marzo de 2017). Dirección de Impuestos y Aduanas Nacionales de Colombia. La Dirección de Impuestos y Aduanas Nacionales. Obtenido de Reconocimiento Mutuo Paises Alianza Del Pacifico: https://www.dian.gov.co/ aduanas/oea/inicio/Documents/Avan-
ces\%20Acuerdo\%20de\%20Reconocimiento\%20Mutuo\%20de\%20los\%20 pa\%C3\%ADses\%20de\%20la\%20Alianza\%20del\%20Pacifico.pdf

EKOS. (30 de junio de 2015). Certificación: un pase hacia la excelencia Un objetivo empresarial. Obtenido de https://www. ekosnegocios.com/articulo/certificacionun-pase-hacia-la-excelencia-un-objetivoempresarial

Guzmán, E. B. (2016). Análisis De Los Operadores Económicos Autorizados Y Su Incidencia En El Comercio Exterior. Guayaquil, Ecuador.

Inter-American Development Bank. (julio de 2018). http://conexionintal.iadb.org. Obtenido de http://conexionintal.iadb.org/ wp-content/themes/mobius/versionimpresionsingle.php?articleld $=12120$

Malhotra, N. K. (2008). Investigacion De Mercados Un Enfoque Aplicado. Mexico: PEARSON EDUCACIÓN.

Ministerio de Comercio Exterior. (16 de octubre de 2018). Pleno De La Asamblea Nacional Aprobó Acuerdo Sobre Facilitación Del Comercio. Obtenido de https://www.comercioexterior.gob.ec/pleno-de-la-asambleanacional-aprobo-acuerdo-sobre-facilitacion-del-comercio/

Miranda, F., Chamorro, A., y Rubio, S. (01 de enero de 2017). Clarificando el concepto de certificación. Clarificando el concepto de certificación: El caso español , 2. España. Obtenido de http:// merkado.unex.es/operaciones/descargas/Certificaci\%C3\%B3n\%20en\%20 Espa\%C3\%B1a.pdf

Moran Espin, D. F. (2014). Análisis sobre el impacto del nuevo sistema Ecuapass en los operadores de comercio exterior del Ecuador. Quito. Obtenido de http://repositorio.ute. edu.ec/handle/123456789/8385

Organización Mundial de Aduanas. (2017). Recueil sur les Programmes d' Opérateurs économiques agréés. Bruselas: Organización Mundial de Aduanas. Obtenido de http://dernegocios.uexternado.edu.co/negociacion/ el-operador-economico-autorizado-oea/

Organización Mundial de Aduanas. (junio de 2018). Marco De Normas Safe. Obteni- 
do de http://www.wcoomd.org/-/media/ wco/public/es/pdf/topics/facilitation/ instruments-and-tools/tools/safe-package/safe-framework.pdf?la=en

Secretaría Nacional de Planificación y Desarrollo. (01 de enero de 2017). Plan Nacional de Desarrollo 2017-2021-Toda una Vida. Obtenido de http://www.planificacion. gob.ec/wp-content/uploads/downloads/2017/10/PNBV-26-OCT-FINAL_OK. compressed1.pdf

SENAE. (29 de diciembre de 2017). SENAE-SENAE2017-0693-RE. Recuperado el 25 de 11 de 2019, de https://www.aduana.gob.ec/resolucion/senae-senae-2017-0693-re-2/

SENAE. (1 de enero de 2018). Decreto 312. Obtenido de https://www.aduana.gob.ec/ wp-content/uploads/2018/03/Decreto_ No._312.pdf

SENAE. (4 de noviembre de 2019). aduana.gob.ec. Recuperado el 4 de 11 de 2019, de aduana. gob.ec: https://www.aduana.gob.ec/oea/ noticias-oea/

SENAE. (27 de mayo de 2019). Ecuador Firma Acuerdo De Reconocimiento Mutuo con la Comunidad Andina. Obtenido de https://www.aduana.gob.ec/wp-content/ uploads/2019/05/BOLETIN-ADUANA-FIRMA-ACUERDO-DE-RECONOCIMIENTO-MUTUO-CON-LA-CAN.pdf

Sistema Económico Latinoamericano y del Caribe. (mayo de 2012). Mecanismos y modalidades para fomentar el comercio entre los países de América del Sur. Obtenido de http://www.sela.org: http://www.sela. org/media/265675/t0236000049920-mecanismos_modalidades_fomentar_ comercio_paises_america_sur.pdf

SUNAT Perú. (10 de diciembre de 2017). http:// oea.sunat.gob.pe. Recuperado el 26 de noviembre de 2019, de http://oea.sunat. gob.pe/docs/beneficios-oea.pdf 